\title{
Comparison between the International Physical Activity Questionnaire and the American College of Sports Medicine/American Heart Association criteria to classify the physical activity profile in adults ${ }^{1}$
}

\author{
Suzana Alves de Moraes² \\ Cláudio Shigueki Suzuki ${ }^{3}$ \\ Isabel Cristina Martins de Freitas ${ }^{4}$
}

\begin{abstract}
Objective: the study aims to evaluate the reproducibility between the International Physical Activity Questionnaire and the American College of Sports Medicine/American Heart Association criteria to classify the physical activity profile in an adult population living in Ribeirão Preto, SP, Brazil. Methods: population-based cross-sectional study, including 930 adults of both genders. The reliability was evaluated by Kappa statistics, estimated according to socio-demographic strata. Results: the kappa estimates showed good agreement between the two criteria in all strata. However, higher prevalence of "actives" was found by using the American College of Sports Medicine/American Heart Association. Conclusions: although the estimates have indicated good agreement, the findings suggest caution in choosing the criteria to classify physical activity profile mainly when "walking" is the main modality of physical activity.
\end{abstract}

Descriptors: Motor Activity; Reproducibility of Results; Cross-Sectional Studies.

\footnotetext{
${ }^{1}$ Supported by Conselho Nacional de Desenvolvimento Científico e Tecnológico (CNPq), process \# 505622/2004-1.

${ }^{2}$ PhD, Associate Professor, Escola de Enfermagem de Ribeirão Preto, Universidade de São Paulo, WHO Collaborating Centre for Nursing Research Development, Ribeirão Preto, SP, Brazil.

${ }^{3}$ PhD, Adjunct Professor, Departamento de Educação Física, Universidade Estadual do Centro-Oeste, Irati, PR, Brazil. Scholarship holder from Conselho Nacional de Desenvolvimento Científico e Tecnológico (CNPq).

${ }^{4}$ PhD, Social Scientist. Scholarship holder from Conselho Nacional de Desenvolvimento Científico e Tecnológico (CNPq).
}

Corresponding Author:

Suzana Alves de Moraes

Universidade de São Paulo. Escola de Enfermagem de Ribeirão Preto

Departamento Materno-Infantil e Saúde Pública

Av. Bandeirantes, 3900

Bairro: Monte Alegre

CEP: 14040-902, Ribeirão Preto, SP, Brasil

E-mail: samoraes@usp.br 


\section{Introduction}

The assessment of the physical activity profile (PAP) in population-based studies has aroused interest in recent decades. Questionnaires are used as the main tool to measure it, in view of their low cost, agile application and easy data collection. In view of the excess information for this purpose, however, which was not always comparable and originated in different kinds of questionnaires, an international group of researchers, with support from the World Health Organization (WHO), elaborated the International Physical Activity Questionnaire (IPAQ), which was standardized and validated in different parts of the world, including Brazil(1-2).

The appropriate definition of the PAP in people from a given reference population is fundamental in Epidemiology and Public Health, considering evidence of associations between certain profiles and sociodemographic, behavioral and health-related factors ${ }^{(3-4)}$.

The WHO's proposal when elaborating the IPAQ(1) was based on the standardization of the criteria used to verify physical exercise levels, thus permitting the comparison of their respective prevalence rates and the investigation of associated factors in different populations and countries.

The IPAQ(1) offers an additional advantage to most questionnaires aimed at measuring PAP, which is the quantification of the metabolic rate, defined as the quantity of METs (Metabolic Equivalent of Task) in minutes per week, for each type of activity, using the information in the Ainsworth Compendium(5-6). In other words, the algorithm proposed in the IPAQ ${ }^{(1)}$ to define physical exercise levels includes not only the frequency, intensity and duration of the exercise, but also the metabolic rate attributed to the activity type, which by itself makes this instrument more complete than most instruments used for this purpose in field research.

Although the IPAQ Questionnaire ${ }^{(1)}$ has been used in population based research, however, different criteria have been applied to classify the participants. While some studies have adopted the criteria proposed in the IPAQ Protocol(1-6), others have chosen the classification proposed by the American College of Sports Medicine/ American Heart Association (ACSM/AHA)(7), sometimes hampering the comparison of results from Brazilian or international studies.

In view of the above, this study was aimed at estimating the prevalence rates of physical activity in adults who participated in the OBEDIARP Project, which were verified according to the criteria of the IPAQ Protocol and those recommended by ACSM/AHA, assessing the reproducibility between the two protocols' criteria.

\section{Methods}

The data derived from a population-based epidemiological study (Risk factors for overweight, obesity and diabetes mellitus in Ribeirão Preto, SP, 2006-OBEDIARP Project). The sampling process was developed in three stages and the precision of the estimates, calculated in a sample of 1,205 individuals, corresponded to sampling errors set at approximately $2 \%$ (for prevalence rates inferior to $15 \%$ or superior to $75 \%$ ) and $3 \%$ (for prevalence rates between $20 \%$ and $80 \%)^{(8)}$. According to estimates from the 2000 Population Census ${ }^{(9)}$, the city's census tracts served as the Primary Sampling Unit ( $n=81$ tracts drafted), while the households $(n=1,671)$ and residents $(n=1,205)$ corresponded to the units drafted in the second and third stages, respectively. In the third stage, one person aged 30 years or older was drafted among the $(N)$ residents in the households drafted, excluding pregnant women or women up to the sixth month after birth (selection criterion). In total, 930 participants were interviewed, male and female, aged 30 years or older, living in urban Ribeirão Preto in 2006. The response rate corresponded to $78 \%$ and the losses were distributed as follows: $6.7 \%$ due to address change, $0.6 \%$ due to death and $14.0 \%$ to refusal, the latter after six attempts to recover the interviews, on different days and times, throughout the week. The variability that was introduced, mainly in the third sample fraction, was corrected through the introduction of sample weights, equal to the number of eligible units at each household, resulting in a weighted sample $\left(n_{w}\right)$ of 2,197 participants.

The information was collected through home interviews, applied by trained and calibrated interviewers $^{(10)}$, with the help of structured questionnaires. Details about the sample characteristics of the OBEDIARP Project are available in an earlier publication ${ }^{(11)}$.

To assess the PAP, the IPAQ Questionnaire-short version $^{(1-6)}$ was used. The participants were classified in three categories: a) moderate activity: 05 or more days per week of any combination between walking, and moderate or vigorous-intensity activity, accumulating at least 600 METs*minutes* week $^{-1}$; b) high activity: 07 or 
more days of any combination between these activities,

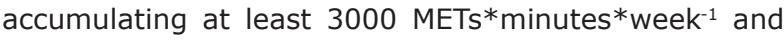
c) insufficient activity: participants not classified in any of the above categories. For the purpose of the present study, the participants classified under: "moderate activity" and "high activity" were grouped in a sole category, called "sufficient activity", which together with "insufficient activity" represented the two study categories for PAP classification.

As recommended by the IPAQ ${ }^{(1-6)}$ protocol, the definition of PAP includes not only the frequency, duration and intensity of exercises, but also the metabolic rate deriving from the activity, which makes the instrument stricter and more specific to classify the subjects. To calculate the metabolic rate, defined as the amount of Mets in minutes per week, each type of exercise was classified according to the recommendations found at the Physical Activities Compendium( ${ }^{(5-6)}$. Its authors defined the MET as the ratio between two rates: the energy spent to perform a given activity (numerator) and the metabolic waste at resting (denominator), the latter equivalent to $1.0(4.184 \mathrm{~kJ}) \mathrm{kg}^{-1} \mathrm{~h}^{-1}$, where $\mathrm{kJ}=$ energy in kilojoules; $\mathrm{kg}=$ kilo of body weight and $\mathrm{h}=$ hour.

To respond to the study objective, the participants were also ranked according to the ACSM/AHA(7) criteria. Individuals who combined walking, moderate or vigorous-intensity exercises for at least 150 minutes in five days per week, or who practiced at least 20 minutes of vigorous-intensity exercise at least three days per week were classified as "sufficiently active", while those who did not meet any of these criteria were considered "insufficiently active".
Point and 95\% confidence intervals for the prevalence rates of physical activity were estimated according to gender and age range groups, in a weighted sample of 2,197 participants $\left(n_{w}\right)$, considering the sample design effect ( deff $=1.0843$ ). The reproducibility between the IPAQ ${ }^{(1-6)}$ and $\mathrm{ACSM} / \mathrm{AHA}^{(7)}$ criteria was measured using Kappa statistics ${ }^{(12-13)}$, also estimated by points and $95 \%$ confidence intervals, according to sociodemographic categories, considering: gender (male and female); age, classified in ten-year intervals; education, classified in four categories, according to the number of complete years of formal education, without any fails ("<1"; "1-3"; "4-7" and " $\geq 8$ years") and individual income in Reais ( $\mathrm{R} \$)$, using the month before the interview as a reference. Those participants who did not declare any income were classified as "no income", while those who did were classified in three categories, according to the cut-offs equivalent to the distribution tertiles ("< R\$ 520"; "R\$ 520-1100" and "> R\$ 1100).

The OBEDIARP Project received approval from the Research Ethics Committee at the University of São Paulo at Ribeirão Preto College of Nursing (Process 0528/2005). All participants signed an Informed Consent Form, in compliance with the National Health Council Resolution 196/96.

\section{Results}

The prevalence rates of physical activity according to gender and age groups, considering the IPAQ and ACSM/AHA criteria are displayed in Tables 1 and 2, respectively.

Table 1 - Prevalence of physical activity, according to gender and age, with the respective confidence interval (95\%), using the International Physical Activity Questionnaire criteria. Ribeirão Preto, SP, Brazil, 2006

\begin{tabular}{|c|c|c|c|c|c|c|c|c|}
\hline \multirow{3}{*}{ Variables } & \multicolumn{4}{|c|}{ Male } & \multicolumn{4}{|c|}{ Female } \\
\hline & \multicolumn{2}{|r|}{ Sufficient } & \multicolumn{2}{|c|}{ Insufficient } & \multicolumn{2}{|c|}{ Sufficient } & \multicolumn{2}{|c|}{ Insufficient } \\
\hline & $\mathbf{N}_{\mathrm{w}}{ }^{*}$ & $\% *\left[\mathrm{Cl}_{95 \%}\right]$ & $\mathbf{N}_{\mathrm{w}}{ }^{*}$ & $\% *\left[\mathrm{Cl}_{95 \%}\right]$ & $\mathbf{N}_{\mathrm{w}}{ }^{*}$ & $\% *\left[\mathrm{Cl}_{95 \%}\right]$ & $\mathbf{N}_{\mathrm{w}}{ }^{*}$ & $\% *\left[\mathrm{Cl}_{95 \%}\right]$ \\
\hline \multicolumn{9}{|l|}{ Age ranges } \\
\hline $30-39$ years & 99.9 & $40.6[30.6-51.3]$ & 146.4 & 59.4 [48.6-69.3] & 151.4 & 37.4 [29.4-46.1] & 253.2 & $62.6[53.8-70.5]$ \\
\hline $40-49$ years & 45.7 & $28.9[19.3-40.7]$ & 112.4 & 71.1 [59.2-80.6] & 141.5 & 27.9 [21.5-35.3] & 365.4 & 72.1 [64.6-78.5] \\
\hline $50-59$ years & 51.9 & $42.2[29.3-56.1]$ & 71.1 & 57.8 [43.8-70.6] & 124.3 & 37.8 [28.7-47.8] & 204.3 & $62.2[52.1-71.2]$ \\
\hline$\geq 60$ years & 46.8 & 37.9 [24.8-52.8] & 76.7 & 62.1 [47.1-75.1] & 75.8 & 25.6 [17.7-35.2] & 220.9 & $74.4[64.7-82.2]$ \\
\hline Total & 244.4 & $37.5[30.9-44.6]$ & 406.7 & 62.5 [55.4-69.0] & 493.1 & 32.1 [27.4-37.0] & 1044 & 67.9 [62.9-72.5] \\
\hline
\end{tabular}

* Weighted estimates, considering the design effect.

As observed, the prevalence rates of sufficient practice were higher when adopting the ACSM/AHA criteria (Table 2 ) in comparison with the IPAQ criteria
(Table 1), mainly among women and participants aged 60 years or older. 
Table 2 - Prevalence of physical activity, according to gender and age, with the respective confidence intervals (95\%), using the American College of Sports Medicine/American Heart Association criteria. Ribeirão Preto, SP, Brazil, 2006

\begin{tabular}{|c|c|c|c|c|c|c|c|c|}
\hline \multirow{3}{*}{ Variables } & \multicolumn{4}{|c|}{ Male } & \multicolumn{4}{|c|}{ Female } \\
\hline & \multicolumn{2}{|r|}{ Sufficient } & \multicolumn{2}{|c|}{ Insufficient } & \multicolumn{2}{|r|}{ Sufficient } & \multicolumn{2}{|c|}{ Insufficient } \\
\hline & $\mathbf{N}_{\mathrm{w}}{ }^{*}$ & $\left.\% * \mathrm{Cl}_{95 \%}\right]$ & $\mathbf{N}_{\mathrm{w}}{ }^{*}$ & $\% *\left[\mathrm{Cl}_{95 \%}\right]$ & $\mathbf{N}_{\mathrm{w}}{ }^{*}$ & $\% *\left[\mathrm{Cl}_{95 \%}\right]$ & $\mathbf{N}_{\mathrm{w}}{ }^{*}$ & $\% *\left[\mathrm{Cl}_{95 \%}\right]$ \\
\hline \multicolumn{9}{|l|}{ Age ranges } \\
\hline $30-39$ years & 100.1 & $40.7[30.9-51.2]$ & 146.2 & 59.4 [48.8-69.1] & 160.6 & 39.7 [31.8-48.2] & 244.1 & $60.3[51.9-68.2]$ \\
\hline 40-49 years & 50.3 & $31.8[22.0-43.6]$ & 107.8 & $68.2[56.4-78.0]$ & 154.2 & $30.4[24.1-37.6]$ & 352.7 & $69.6[62.4-75.9]$ \\
\hline $50-59$ years & 52.0 & $42.2[29.4-56.2]$ & 71.2 & 57.8 [43.8-70.6] & 126.7 & 38.6 [29.5-48.5] & 201.8 & $61.4[51.5-70.5]$ \\
\hline$\geq 60$ years & 54.3 & $43.9[30.4-58.4]$ & 69.3 & 56.1 [41.6-69.6] & 90.4 & 30.5 [22.8-39.5] & 206.3 & $69.5[60.5-77.3]$ \\
\hline Total & 256.7 & $39.4[33.0-46.2]$ & 394.4 & $60.6[53.8-67.0]$ & 532 & 34.6 [30.3-39.3] & 1005 & 65.4 [60.8-69.8] \\
\hline
\end{tabular}

* Weighted estimates, considering the design effect.

In Table 3, the reproducibility between the IPAQ and ACSM/AHA criteria related to physical activity was good/excellent in both gender, including the crude and specific estimates, the later one corresponding to those for sociodemographic variables (Kappa>0.70).

Table 3 - Kappa statistics according to sociodemographic variables with respective confidence intervals (95\%) to assess the agreement between the two criteria: International Physical Activity Questionnaire and American College of Sports Medicine/American Heart Association. Ribeirão Preto, SP, Brazil, 2006

\begin{tabular}{|c|c|c|c|c|}
\hline \multirow{2}{*}{ Variable } & \multicolumn{2}{|c|}{ Male } & \multicolumn{2}{|c|}{ Female } \\
\hline & Kappa & Cl $95 \%$ & Kappa & CI $95 \%$ \\
\hline \multicolumn{5}{|l|}{ Age range (years) } \\
\hline $30-39$ & 0.96 & $0.77-1.15$ & 0.93 & $0.78-1.08$ \\
\hline $40-49$ & 0.93 & $0.70-1.17$ & 0.93 & $0.80-1.06$ \\
\hline $50-59$ & 1.00 & $0.73-1.27$ & 0.99 & $0.82-1.15$ \\
\hline$\geq 60$ & 0.87 & $0.60-1.15$ & 0.88 & $0.70-1.06$ \\
\hline \multicolumn{5}{|l|}{ Educational Level } \\
\hline Illiterate, $<1$ year & 1.00 & $0.41-1.59$ & 0.72 & $0.34-1.09$ \\
\hline $1-3$ years & 0.92 & $0.57-1.28$ & 0.94 & $0.73-1.15$ \\
\hline 4-7 years & 0.96 & $0.77-1.19$ & 0.97 & $0.83-1.10$ \\
\hline$\geq 8$ & 0.93 & $0.77-1.09$ & 0.93 & $0.82-1.03$ \\
\hline \multicolumn{5}{|l|}{ Income in Reais (thirds) } \\
\hline No income & 0.96 & $0.70-1.23$ & 0.92 & $0.81-1.04$ \\
\hline$<520.00$ & 0.92 & $0.54-1.30$ & 0.96 & $0.81-1.11$ \\
\hline $520.00-1,100.00$ & 0.94 & $0.73-1.13$ & 0.91 & $0.71-1.10$ \\
\hline$>1,100.00$ & 0.96 & $0.76-1.15$ & 0.94 & $0.74-1.14$ \\
\hline Crude Kappa & 0,95 & $0.83-1.06$ & 0.93 & $0.85-1.01$ \\
\hline
\end{tabular}

\section{Discussion}

The reproducibility between the IPAQ(1-6) and ACSM/ $\mathrm{AHA}^{(7)}$ criteria, expressed through the estimated Kappa statistics, was satisfactory. Kappa coefficients between 0.81 and 1.00 indicate "almost perfect" agreement ${ }^{(12)}$. In this study, reproducibility indicators were chosen instead of validation indicators (sensitivity, specificity and predictive values), keeping in mind that none of both criteria can be considered as "gold standard"(13). Regardless of good reproducibility between the two criteria, the prevalence rates of sufficient practice were overestimated when using the criterion recommended by $\mathrm{ACSM} / \mathrm{AHA}^{(7)}$.

The fact that the IPAQ protocol estimates the metabolic rate, beyond the duration, frequency and intensity of the activity, was certainly the main reason for the respective differences in the prevalence rates of physical activity according to the two criteria. Another important consideration is that, besides permitting the identification of PAP, the IPAQ Protocol(1-6) includes the measurement of sitting time during the week, an 
important indicator of sedentary behavior and also associated with risk or protection for chronic outcomes ${ }^{(4)}$.

In exploratory analyses developed as part of the present study, it was identified that, in the calculation of the crude Kappa, the pairs of disagreement between the two criteria $(n=24)$ corresponded to the participants for whom walking was the main physical activity modality. As these participants did not reach the minimum 600 METs*min*week ${ }^{-1}$ through walking only, they were classified as "insufficiently active" according to the IPAQ ${ }^{(1-6)}$, but as "sufficiently active" when adopting the ACSM/AHA(7) criteria. In addition, among the participants who corresponded to the disagreement pairs, walking represented $84.2 \%$ of the total time (minutes/week) spent on physical activity and $73 \%$ of the total metabolic rate, expressed as METs*min*week ${ }^{-1}$.

Although the disagreement pairs contributed to just a small drop in the agreement levels between the two criteria, the prevalence rates of sufficient practice were overestimated when applying the ACSM/AHA criterion(7). Therefore, it is highlighted that this criterion can expand the spectrum of false-positive individuals, which by itself represent a PAP classification error and could also reflect in spurious associations when considering physical activity as an exposure factor or as an outcome in the analytic association studies.

In the Inquérito de Saúde do Estado de São Paulo $\left(\right.$ ISA-SP)(14), the authors used the ACSM/AHA ${ }^{(7)}$ criteria to identify the prevalence of physical activity in a sample of 1,950 participants aged 60 years or older and found a prevalence rate of "sufficiently active" corresponding to $67.7 \%$ among men and $78.5 \%$ among women. These results are almost twice the rate found among men and thrice the rate found among women in the present study for adults aged 60 years or older (37.9\% and $25.6 \%$, respectively). The increased prevalence rates of physical activity in the ISA-SP(14) seems to confirm the hypothesis that the application of the ACSM/AHA ${ }^{(7)}$ criterion can overestimate the prevalence of "sufficiently active" individuals, considering, on the one hand, the non-inclusion of the metabolic rate for physical activity, and on the other the fact that, as a rule, walking is the main modality in the elderly population.

\section{Conclusion}

Based on the present study findings, it is concluded that, although both - IPAQ and ACSM/AHA - are reproducible (Kappa $\approx 1$ ), they are not equivalent regarding the prevalence of physical activity, in adults.
Therefore, caution is due when interpreting research results in which the ACSM/AHA was used, which can overestimate the prevalence of sufficient practice, when compared to the IPAQ, mainly when walking is the main modality. On the other hand, overestimated prevalence rates of physical activity do not only lead to a mistaken population diagnosis, but also can delay the implementation of programs to promote healthy habits in the adult population.

\section{References}

1. Craig C, Marshall A, Sjostrom M, Bauman AE, Booth $M L$, Pratt $M$, et al. International Physical Questionnaire: 12-country reliability and validity. Med Sci Sports Exercise. 2003; 35:1381-95.

2. Matsudo S, Araújo, T, Matsudo V. Questionário Internacional de Atividade Física (IPAQ): Estudo de validade e reprodutibilidade no Brasil. Rev Bras Atividade Física e Saúde. 2001;6:5-18.

3. Suzuki CS, Moraes SA, Freitas ICM. Atividade física e fatores associados em adultos residentes em Ribeirão Preto, SP. Rev Saúde Pública. 2011;45(2):311-20.

4.Suzuki CS, Moraes SA, Freitas ICM. Média diária de tempo sentado e fatores associados em adultos residentes no município de Ribeirão Preto-SP, 2006: Projeto OBEDIARP. Rev Bras Epidemiol. 2010;13(4):699-712.

5.Ainsworth BE, Haskell WL, Whitt MC, Irwin ML, Swartz AM, Strath SJ, et al. Compendium of Physical Activities: an update of activity codes and MET intensities. Med Sci Sports Exerc. 2000;32(suppl 9):s498-516.

6.Guidelines for data processing and analysis of the International Physical Activity Questionnaire (IPAQ): short and long forms. 2005. [acesso $15 \mathrm{dez} 2010$ ]. Disponível em: http://www.ipaq.ki.se/scoring.pdf.

7. Haskell WL, Lee IM, Pate RR, Powell KE, Blair SN, Franklin BA, et al. Physical activity and public health: updated recommendation for adults from the American College of Sports Medicine and the American Heart Association. Med Sci Sports Exerc. 2007;39(8):1423-34. 8.Silva NN. Amostragem probabilística: um curso introdutório. 2a ed. São Paulo: Edusp; 2001.

9. Instituto Brasileiro de Geografia e Estatística. Censo Demográfico 2000. Rio de Janeiro; 2000.

10. Castro V, Moraes SA, Mondini L, Freitas ICM. Variabilidade na aferição de medidas antropométricas: comparação de dois métodos estatísticos para avaliar a calibração de entrevistadores. Rev Bras Epidemiol. 2008; 11:278-86. 
11. Moraes SA, Freitas ICM, Gimeno SGA, Mondini L. Prevalência de diabetes mellitus e identificação de fatores associados em adultos residentes em área urbana de Ribeirão Preto, São Paulo,Brasil, 2006: Projeto OBEDIARP. Cad Saúde Pública. 2010;26(5):929-41.

12. Vieira AJ, Garret JM. Understanding interobserver agreement: The Kappa Statistic Fam Med. 2005;37(5):360-3.

13. Maclure M, Willett WC. Misinterpretation and misuse of the Kappa statistic. Am J Epidemiol. 1987;126:161-9. 14. Zaitune MPA, Barros MBA, César CLG, Carandina $L$, Goldbaum M, Alves MCGP. Factors associated with global and leisure-time physical activity in the elderly: a health survey in São Paulo (ISA-SP), Brazil. Cad Saúde Pública. 2010;26(8):1606-18. 